
allemande

\title{
La peur comme moteur de l'action politique
}

Discours et contre-discours sur le DDT aux États-Unis et en République fédérale d'Allemagne dans l'après-guerre

\section{Annette Lensing}

\section{(2) OpenEdition}

1 Journals

Édition électronique

URL : https://journals.openedition.org/allemagne/2238

DOI : $10.4000 / a l l e m a g n e .2238$

ISSN : 2605-7913

Éditeur

Société d'études allemandes

Édition imprimée

Date de publication : 1 juillet 2020

Pagination : 81-97

ISSN : 0035-0974

\section{Référence électronique}

Annette Lensing, "La peur comme moteur de l'action politique », Revue d'Allemagne et des pays de langue allemande [En ligne], 52-1 | 2020, mis en ligne le 06 juillet 2021, consulté le 18 février 2022. 


\section{La peur comme moteur de l'action politique Discours et contre-discours sur le DDT aux États-Unis et en République fédérale d'Allemagne dans l'après-guerre}

\section{- Annette Lensing*}

«Je veux que vous paniquiez. Je veux que vous sentiez la peur que je ressens tous les jours. Je veux que vous agissiez. Je veux que vous agissiez comme vous le feriez en cas de crise. Je veux que vous agissiez comme si la maison était en feu, car elle l'est ${ }^{(1)}$.

C'est en ces termes que la jeune activiste du climat Greta Thunberg s'est adressée aux leaders économiques et politiques réunis à Davos début 2019: par opposition radicale à l'impavidité des décideurs mondiaux, la peur serait l'unique arme permettant d'affronter la catastrophe climatique. Porté par la jeune génération et dirigé contre l'attentisme des aînés auxquels il renvoie un miroir culpabilisateur, cet appel retentit avec force, à un moment où l'humanité, en prise avec des problèmes environnementaux intenses et récurrents, semble particulièrement vulnérable. À une époque où la désinformation prospère à mesure que l'influence des médias établis diminue, les activistes climatiques font l'objet d'attaques répétées, portées notamment par les médias conservateurs et la "fachosphère», qui les accusent de nourrir une fascination irrationnelle pour la catastrophe et de méconnaître les «vrais" problèmes de leur temps (chômage, crise migratoire, etc.).

Amplifiées à l'ère du numérique et de la démultiplication des sources d'information, ces réactions rappellent celles que suscitèrent les ouvrages d'alerte environnementale des années 1960-1970. Issus de la plume de «survivalistes ${ }^{(2)}$, des intellectuels

* Maîtresse de conférences en civilisation allemande, Université de Caen Normandie, membre de l'ERLIS.

1 «I want you to panic. I want you to feel the fear I feel every day. And then I want you to act. I want you to act as you would in a crisis. I want you to act as if the house is on fire. Because it is.» Greta THUNBERG, World Economic Forum, Davos, 25.01.2019, www.fridaysforfuture.org/greta-speeches\#greta speech_jan25_2019 (consulté le 01.08.2019).

2 Sur le survivalisme, voir John S. Dryzek, The Politics of the Earth. Environmental Discourses, Oxford, Oxford University Press, 2013. 
et scientifiques anglo-saxons et américains pour la plupart, ils exprimaient la peur que les désastres écologiques observables à l'échelle globale ne précipitent la fin de la civilisation. Printemps silencieux, publié en 1962 par la biologiste américaine Rachel Carson (1907-1964) et considéré comme l'un des déclencheurs de la «révolution écologique» aux États-Unis, en offre un exemple ${ }^{(3)}$. Mettant en garde contre les dangers des pesticides, cet ouvrage est, encore aujourd'hui, contesté. Carson déclencha l'ire de scientifiques, de représentants politiques et d'industriels qui l'attaquaient sur son sexe, ses compétences et les faits qu'elle avançait. S'érigeant contre une hystérie catastrophiste qui irait à l'encontre de toute objectivité scientifique, ils soufflaient le chaud et le froid sur les peurs anciennes qui hantaient la société - peur de la famine, de la dépression économique, des maladies et épidémies, etc. - et d’angoisses nouvelles qui se nourrissaient en premier lieu de la peur de l'apocalypse nucléaire. C'est à ces émotions et à leur ancrage dans un temps et une culture donnés qu'est voué cet article. En quoi l'étude des discours sur le DDT - tant celui de ses défenseurs que celui de ses détracteurs - permet-elle d'interroger la globalisation des peurs pendant l'«âge de l'anxiété " ${ }^{(4)}$ ? Sur la base d'analyses textuelles et iconographiques, cet article présentera le contexte et les enjeux de la parution de Printemps silencieux pour interroger plus particulièrement le discours de crise qu'il véhicule, avant d'éclairer sa réception dans l'espace médiatique ouest-allemand des années 1960.

\section{Contexte et enjeux de Printemps silencieux}

«Le Printemps silencieux est arrivé comme un cri dans le désert, un plaidoyer absolument sincère, solidement étayé et brillamment écrit, qui a changé le cours de l'histoire. Sans ce livre, le mouvement écologiste aurait pu être largement retardé - ou tout simplement ne jamais voir le jour ${ }^{(5)}$.

C'est en ces termes élogieux qu'Al Gore présenta le bestseller de Carson dans l'introduction à sa réédition en 1994: le démocrate américain s'y posait en héritier du combat de celle qui lui aurait inspiré la rédaction de Sauver la planète: l'écologie et l'esprit humain (1993) et dont la photo trônait dans son bureau, parmi les leaders du monde politique $^{(6)}$. Ce témoignage illustre l'impact des ouvrages d'alerte environnementale des années 1960-1970, leur effet d'entraînement pour des hommes et des femmes qui s'en inspirèrent pour former le «panthéon intellectuel de l'écologie politique » ${ }^{(7)}$.

3 Cf. Rachel Carson, Silent Spring, Londres, Penguin Classic, 1962. Robert Downs présente Silent Spring comme le livre ayant lancé le mouvement environnemental moderne. Cf. Robert B. Downs, Books That Changed America, New York, Macmillan, 1974. Cet article s'appuie sur la version française de l'ouvrage: Rachel Carson, Printemps silencieux, Marseille, Wildproject, 2019 (3éd.). Cf. Jean-Jacques Courtine, «La peur, à l'âge de l'anxiété», in: Alain Corbin, Jean-Jacques Courtine et al., Histoire des émotions, t. 3 : De la fin du XIX siècle à nos jours, Paris, Seuil, 2017, p. 307-325.

5 Al Gore, «Introduction», in: R. CARson, Printemps silencieux (note 3), p. 25-39, ici p. 25.

6 Ibid., p. 29.

7 Anna Trespeuch-Berthelot, «La réception des ouvrages d'alerte environnementale dans les médias français (1948-1973)", Le Temps des médias, n² 25, automne 2015, p. 104-119, www.cairn.info/revuele-temps-des-medias-2015-2-page-104.htm (consulté le 01.08.2019). 
«Du poison au supermarché» ${ }^{(8)}$.

\section{Les promesses de l'industrie chimique dans l'après-guerre}

Avec Printemps silencieux, Carson voulait mettre ses contemporain.e.s en garde contre les dangers directs et indirects de l'utilisation massive des pesticides, plus particulièrement de la famille des organochlorés, parmi lesquels le dichlorodiphényltrichloroéthane $(\mathrm{DDT})^{(9)}$. Le terme générique de "pesticide» vient du latin "caedere», tuer, et "pestis», maladie contagieuse, et désigne communément des substances chimiques dont le but est d'exterminer les «ravageurs», les «mauvaises herbes» et les insectes considérés comme "nuisibles», afin de diminuer les pertes, d'augmenter les rendements et de réduire la propagation de maladies. À travers le DDT, la biologiste s'attaquait à une industrie chimique en pleine expansion et à une substance pour laquelle le chimiste suisse Paul Hermann Müller, qui avait mis en évidence l'utilité du DDT comme insecticide en 1939, avait obtenu le prix Nobel de médecine en 1948, en raison notamment de son efficacité pour l'éradication d'insectes vecteurs de maladies (paludisme, maladie du sommeil, typhus) ${ }^{(10)}$. Sur la base des avancées de la chimie organique, la synthétisation et l'usage des pesticides de la famille des organochlorés (DDT, aldrine, dieldrine, etc.) connurent un développement important; ils occupèrent une place de premier plan sur le marché des pesticides jusque dans les années 1970: selon les chiffres avancés par Carson, près de cinq cents nouvelles substances chimiques étaient exploitées tous les ans aux ÉtatsUnis au début des années $1960^{(11)}$. Entre 1947 et 1960, la consommation de produits chimiques serait passée de 56 à 289 millions de kilos. La «révolution verte» modifia profondément la nature et l'usage des produits phytosanitaires: inondant le marché, ceux-ci répondaient à l'évolution des pratiques agricoles - marquées notamment par l'introduction de nouvelles plantes, la mécanisation des exploitations et l'intensification de la production ${ }^{(12)}$. Ils portaient la promesse d'éradiquer définitivement la famine et les « révoltes de la faim» qui avaient profondément marqué les consciences des sociétés occidentales.

Dans l'immédiat après-guerre, les publicités pour le DDT jouaient explicitement sur ces peurs, au premier rang desquelles celle du manque de ressources et la peur

8 «Gift aus dem Supermarket», in: «Schädlingsbekämpfung. Schweigen im Walde», Der Spiegel, n 46 (1962), 14.11.1962, p. 122.

9 Dès 1945, Carson proposa au Reader's Digest d'écrire un article sur la toxicité des épandages de DDT; elle décida d'y consacrer un ouvrage après avoir reçu une lettre de son amie Olga Owens Huckins en 1958, l'informant d'oiseaux morts dans son jardin suite à l'épandage de DDT. Cf. Cheryll GLOTfELTy, «Cold War, Silent Spring: The Trope of War in Modern Environmentalism», in: Craig WaddeLL (éd.), And no birds sing. Rhetorical Analyses of Rachel Carson's Silent Spring, Carbondale, Southern Illinois University, 2000, p. 157-168, Appendix Chronology.

10 C'est lors de l'épidémie de typhus à Naples (1942-1943) que le DDT fut pour la première fois largement utilisé. Pendant la Seconde Guerre mondiale, on s'en servit notamment pour l'épouillage des soldats. Cf. C. Glotfelty, «Cold War, Silent Spring» (note 9), p. 158.

11 Cf. R. Carson, Printemps silencieux (note 3), p. 49.

12 Dans les années 2000, l'emploi du terme de "produits phytosanitaires» s'imposa, le terme de «pesticide» ayant pris une connotation négative au fil de la médiatisation des débats sur la toxicité de ces substances. Cf. Nathalie Chèvre, Nelly Niwa, «Phytosanitaires et fertilisants», in: Dominique Bourg, Alain Papaux (éd.), Dictionnaire de la pensée écologique, Paris, PUF, 2015, p. 769-772. 
des épidémies, tout en mettant en avant les «remèdes» que l'industrie chimique préconisait: elles suivaient une ligne d'argumentation binaire fondée sur des couples antithétiques (guerre/paix, santé/maladie, manque/profusion) clairement identifiables par le consommateur - et en l'occurrence la consommatrice, figure centrale d'une démocratisation de l'achat de pesticides qui, réputés efficaces et peu coûteux, avaient fait leur entrée au supermarché. Dans une publicité de l'entreprise Pennsalt Chemicals parue dans le Time Magazine en 1947, on voit une femme entonner la mélodie "DDT is good for m-e-e-e», en chœur avec vache, chien, poule, pomme et pomme-de-terre ${ }^{(13)}$. En tant que «bienfaiteur pour toute l'humanité» qui « [élimine] une armée d'insectes nuisibles », le DDT accroîtrait le confort, la santé et la sécurité, ce que détaillent six vignettes à grand renfort de superlatifs: le DDT rendrait les vaches plus charnues, les pommes plus juteuses et le foyer plus sain. Tout en exprimant la foi forte dans le progrès technique et scientifique, cette publicité réactualise la vision romantisée d'une nature préindustrielle, placée sous le double signe de la fuite hors du temps et du retour à une relation harmonieuse entre l'être humain et la nature. Soulignant son efficacité, les publicités insistaient aussi sur l'innocuité du DDT qui était explicitement recommandé pour être utilisé dans les chambres d'enfant: une publicité pour le papier peint Trimz montre ainsi un nourrisson au teint frais et sain protégé par l'étreinte rassurante de sa mère qui vient d'appliquer du papier-peint imprégné de DDT sur les murs. L'image illustre le slogan suivant: «Protégez vos enfants contre les insectes porteurs de maladies " ${ }^{(14)}$. La représentation de la femme (blanche et de classe moyenne) comme figure nourricière et protectrice est l'un des poncifs de ces publicités qui véhiculent une vision patriarcale des relations entre les sexes; dans le même temps, les femmes sont dépeintes comme modernes et indépendantes, à l'image de cette mère qui applique elle-même le papier peint que «tout le monde peut utiliser [...] sans aide ou expérience préalable " ${ }^{(15)}$ (sic!) ou d'une jeune femme maquillée qui extermine les insectes sans le moindre effort - «Vous pressez le bouton... les punaises tombent raide mort ${ }^{(16)}$.

Dans ces publicités, l'éradication de ce qui nuit au corps des hommes et, par extension, à la nation revêt une signification positive. Sous-tendus, en ces débuts de Guerre froide, par l'affrontement idéologique entre les deux blocs et la propagation d'une vision binaire du monde, l'imaginaire et la rhétorique guerriers s'inscrivent dans un mode de compréhension de la catastrophe qui se diffuse dans les sociétés occidentales à partir du XIX ${ }^{\mathrm{e}}$ siècle et qui redéfinit les relations entre l'homme et la nature: techniciens et scientifiques sont placés au service de la «lutte contre les forces naturelles et leur maîtrise» qui, «au lieu d'une fatalité subie», sont «de plus en plus perçues

13 Affiche publicitaire en couleur pour les produits à base de DDT de la marque Pennsalt, "DDT Is Good for Me-e-e!», Time Magazine, 30.07.1947, Science History Institute, Philadelphie, digital. sciencehistory.org/works/1831ck18w (consulté le 02.08.2019).

14 Publicité en noir et blanc pour le papier-peint Trimz, «Protect Your Children Against Disease-Carrying Insects!», Women's Day, 6 (1947), Science History Institute, Philadelphie, digital.sciencehistory.org/ works/mg74qm295 (consulté le 02.08.2019).

15 «Anyone can put Trimz Wallpaper up without help or previous experience.» Ibid.

16 «Just Press the Button... Bugs Drop Dead», affiche publicitaire en couleur pour Black Flag's Aerosol Insect Killer, 1940-1945, Science History Institute, Philadelphie, digital.sciencehistory.org/works/ mp48sc895 (consulté le 02.08.2019). 
comme une finalité de l'histoire humaine» ${ }^{(17)}$. C'est contre cette modernité techniciste et industrielle que s'érige Carson quand elle dénonce «la croisade pour un univers chimiquement stérile et délivré d'insectes $[\ldots]{ }^{(18)}$.

\section{Printemps silencieux, une fable scientifique}

Il est difficile de faire rétrospectivement le bilan des chiffres avancés par Carson et d'évaluer l'ampleur de la connaissance des risques sanitaires du DDT par ses contemporain.e.s. Ces risques n’étaient pas débattus dans l'espace public américain du début des années 1960, pas plus qu'ils ne faisaient l'objet d'une politique de prévention: malgré l'existence de lois et d'instances de régulation, on se préoccupait peu des effets possibles de l'usage massif des pesticides sur l'être humain et les autres espèces, et les épandages de pesticides par avion étaient monnaie courante - de même que les essais nucléaires se faisaient à ciel ouvert ${ }^{(19)}$. Le potentiel de toxicité des pesticides était toutefois connu: dès les années 1950, des scientifiques et experts environnementaux avaient alerté des risques liés à l'utilisation massive des produits chimiques, pointant du doigt leur impact sur la contamination des eaux et des sols, la reproduction des oiseaux et la santé humaine. Carson s'appuyait sur ces travaux, avançant quantité de statistiques et de témoignages et adjoignant une quarantaine de pages de références et un index approfondi à son ouvrage. Ayant démissionné de l'administration fédérale américaine en 1952 pour se consacrer à l'écriture d'articles et d'ouvrages, la chercheuse pouvait se prévaloir d'une certaine indépendance, alors qu'elle accusait nombre de ses confrères d'être largement à la solde du lobby industriel. Tout en étant «[...] une démonstration fouillée à l'adresse des scientifiques et du public cultivé des risques sanitaires causés par l'usage de produits chimiques » ${ }^{(20)}$, cet ouvrage devait faire œuvre de vulgarisation scientifique, remédier à l'insouciance et à l'ignorance, contrer le discours des journaux spécialisés et de l'administration publique américaine qui présentaient le DDT comme un produit miracle. «L'obligation de subir» donnait à ses concitoyen.ne.s «le droit de savoir» ${ }^{(21)}$.

Pour capter un auditoire large, la biologiste enchâssa sa démonstration scientifique dans une fable des temps modernes ${ }^{(22)}$ : «Il était une fois une petite ville au cœur de l'Amérique où toute vie semblait vivre en harmonie avec ce qui l'entourait» - c'est

17 Cf. François Walter, «Catastrophes», in: D. Bourg et al. (éd.), Dictionnaire de la pensée écologique (note 12), p. 131-134.

18 Cf. R. Carson, Printemps silencieux (note 3), p. 54.

19 Ce n'est qu'en 1972 que les États-Unis stoppèrent les essais nucléaires atmosphériques, au lendemain de la mobilisation d'activistes écologistes et pacifistes contre les essais à Amchitka, une île au large de l'Alaska, et qui marqua les débuts de Greenpeace.

20 A. Trespeuch-Berthelot, «La réception des ouvrages d'alerte environnementale dans les médias français» (note 7$)$, p. 8.

21 Carson reprend la formule de Jean Rostand («l'obligation de subir nous donne le droit de savoir»). Cf. R. Carson, Printemps silencieux (note 3), p. 56.

22 Le recours à la forme littéraire rappelle le roman Die Waffen nieder! de la pacifiste autrichienne Bertha von Suttner, à laquelle on reprocha également un sentimentalisme exacerbé. Cf. Bertha von SuTTNER, Die Waffen nieder! Eine Lebensgeschichte, Dresde, Pierson, 1907. Sur Suttner, voir notamment MarieClaire Hoock-Demarle, Bertha von Suttner (1843-1914). Amazone de la paix, Villeneuve d'Ascq, Presses universitaires du Septentrion, 2014. 
ainsi que s'ouvre la «Fable pour demain», bref chapitre liminaire de Printemps silencieux, où un «mauvais sort» rompt brutalement l'équilibre paisible et harmonieux d'une ville de province américaine. Jadis féconde et bruyante, la nature est réduite à un silence lourd et mortifère: «Ce fut un printemps sans voix. À l'aube, qui résonnait naguère du chœur des grives, des colombes, des geais, des roitelets et de cent autres chanteurs, plus un son ne se faisait désormais entendre [...]» ${ }^{(23)}$. Symbolisée par les oiseaux devenus aphones, la nature printanière a été empoisonnée par les «élixirs de mort» ${ }^{(24)}$, premier acte d'une sombre tragédie dont Carson se fait la prophétesse, en déclarant: «Un effroyable spectre s'est insinué parmi nous sans que nous nous en rendions compte, et cette tragédie imaginaire pourrait aisément devenir une réalité brutale que nous connaîtrons tous » ${ }^{(25)}$. En envisageant la fin de la civilisation et de toute humanité(26), Carson développe un discours catastrophiste.

\section{Possibilités et limites du catastrophisme comme mode d'action et de mobilisation}

Il n'existe pas de définition univoque du catastrophisme, terme polysémique et controversé. Il pourrait se définir a minima par le fait d'«aborder une situation incertaine en se concentrant sur le pire scénario éventuel», de «décider par convention de tenir ce scénario pour certain en l'absence d'actions empêchant sa réalisation » et de «mobiliser tous les moyens à disposition pour éviter que ce scénario ne se réalise» ${ }^{(27)}$. Cette partie se propose de soumettre Printemps silencieux à cette grille de lecture du catastrophisme et d'en interroger la radicalité, en le mettant en résonance avec la nouvelle The Desolate Year que le grand groupe agronomique Monsanto publia dans le Monsanto Magazine en octobre 1962.

\section{Printemps silencieux, plaidoyer pour un catastrophisme éclairé}

Dans Printemps silencieux, Carson déploie quantité de stratégies pour mobiliser ses concitoyen.ne.s contre la menace de l'autodestruction de l'humanité qui, toute latente qu'elle est, lui semble certaine en l'absence d'une modification profonde des modes de pensée et des pratiques. Parmi les stratégies discursives qu'elle met en place figure l'analogie entre les substances chimiques et le nucléaire qui seraient des "(a)lliés sinistres et méconnus» qui «œuvrent [...] à la modification de la nature même du monde - la nature même de la vie» ${ }^{(28)}$. Reprenant à son compte la verve et la posture guerrières de ceux qu'elle dénonce ${ }^{(29)}$, la biologiste assimile l'homme moderne à un

23 Cf. R. Carson, Printemps silencieux (note 3), p. 44.

24 Ibid., $3^{\mathrm{e}}$ chapitre.

25 Ibid., p. 45.

26 Carson insère sa critique des pesticides dans une réflexion éthique sur le devenir de la civilisation humaine et de ses valeurs, se demandant: «En approuvant un acte capable de causer de telles souffrances à des créatures vivantes, ne sommes-nous pas tous diminués dans notre humanité?» Ibid., p. 139.

27 Olivier GodArD, «Catastrophisme», in: Dictionnaire de la pensée écologique (note 12), p. 134-138, ici p. 135.

28 R. Carson, Printemps silencieux (note 3), p. 47.

29 S'intéressant à la rhétorique guerrière de Carson, Glotfelty voit dans le recours au paradigme de la bipolarité du monde la raison de l'impact qu'eut Carson sur l'environnementalisme moderne, en 
apprenti-sorcier qui «joue avec les atomes» et menace de rendre la planète «impropre à toute vie» ${ }^{(30)}$. Tout en filant la métaphore du poison - ses contemporain.e.s seraient les Borgia des temps modernes ${ }^{(31)}$-, Carson tisse le canevas d'une catastrophe future dont la ville fictive est la préfiguration métonymique:

«En dehors du risque d'extermination de l'humanité par une guerre atomique, le problème crucial de notre époque est donc la contamination de notre environnement par des substances d'une incroyable nocivité - des produits qui s'accumulent dans les tissus des plantes et des animaux, pénètrent même jusque dans les cellules reproductrices, où elles altèrent les éléments qui déterminent l'avenir par le moyen de l'hérédité » ${ }^{(32)}$.

Si Carson utilise la métaphore du poison comme fil rouge de sa démonstration, c'est pour mieux rendre visible le caractère insidieux et latent du risque encouru à l'"âge du poison", au fil d'«innombrables petites expositions, quotidiennes et permanentes [qui] peuvent s'avérer plus dangereuses encore» ${ }^{(33)}$. Chapitre après chapitre, Carson fait en effet le catalogue méticuleux des possibles effets nocifs des pesticides sur l'être humain et son environnement, tout en soulignant que l'inquiétude la plus grande résulte non de leur effet visible et immédiat, mais de leur «effet différé» ${ }^{(34)}$. Pour alerter de risques qui sont en partie inscrits dans un avenir hypothétique, Carson reproduit avec acuité des destinées individuelles: le portrait d'une femme ayant consciencieusement aspergé son sous-sol de produits chimiques avant de mourir d'une leucémie aiguë deux mois plus tard ${ }^{(35)}$ semble être le négatif de la publicité du papier-peint Trimz et préfigure la mort de l'auteure en 1964, des suites d'un cancer. Par la mise en avant des problèmes de «santé environnementale» ${ }^{(36)}$, Carson développe un discours environnemental en premier lieu sanitaire. Celui-ci est fondamentalement politique: l'analogie entre mort individuelle et collective, entre apocalypse chimique et nucléaire s'inscrit dans le contexte de la Guerre froide et plus particulièrement de l'acmé des tensions internationales lors de la crise de Cuba d'octobre 1962 qui projette dans un futur proche l'hypothèse d'une guerre nucléaire. Printemps silencieux paraît la même année que Fallout Shelter - l'un de ces nombreux guides qui proposaient des «kits» censés assurer la survie matérielle et psychique en cas de catastrophe nucléaire ${ }^{(37)}$.

Pour rendre la latence de la menace expérimentable par ses contemporain.e.s, Carson ne recourt toutefois pas à un discours apocalyptique, ce qui s'explique par la démarche qui sous-tend l'ouvrage: elle en appelle à l'éthique des responsables, ne plaidant pas pour l'interdiction totale des pesticides, mais pour leur utilisation plus

mobilisant les émotions qui circulaient dans l'opinion publique. Cf. C. Glotfelty, «Cold War, Silent Spring» (note 9), p. 167.

30 R. CARson, Printemps silencieux (note 3), p. 48-49.

31 Ibid., $11^{\mathrm{e}}$ chapitre.

32 Ibid., p. 50.

33 Ibid., p. 209.

34 Ibid., p. 222.

35 Ibid., p. 259.

36 Ibid., p. 221. Sur le lien entre conscience environnementale et conscience sanitaire, voir aussi la préface de Joachim Radkau à la réédition de la version allemande de l'ouvrage: Rachel CARson, Der stumme Frühling, Munich, C.H. Beck, 2019 (5éd.).

37 Cf. Chuck West, Fallout Shelter, Greenwich (Conn.), Fawcett Publications, 1962. 
raisonnable ${ }^{(38)}$. L'impact - direct et indirect - des activités humaines sur l'environnement est au cœur de sa démonstration : prenant en compte les effets non intentionnels des activités humaines, elle assortit sa vision arcadienne de la nature d'une vision utilitariste proche de ce que sera le développement durable ${ }^{(39)}$. Son discours s'apparente moins à la conjuration fataliste et démobilisatrice de l'apocalypse qu'à l'expression d'une pensée politique pragmatique et en mouvement dont l'ambition est de contribuer à la réflexion sur des alternatives possibles, comme l'introduction de prédateurs naturels ou la stérilisation des mâles ${ }^{(40)}$.

Aux États-Unis, cette alerte tombait sur un terreau particulièrement fertile, l'exploitation des sols et des forêts y étant bien plus avancée qu'en Europe centrale et de l'Ouest. En outre, le DDT comptait parmi ses victimes "collatérales" l'oiseau emblématique du pays, le Bald Eagle ou "aigle à tête blanche", ce qui contribua à émotionaliser le débat outre-Atlantique, en liant fortement dimensions environnementales et nationales ${ }^{(41)}$. La forte charge identitaire du scandale du DDT est l'un des facteurs explicatifs de l'importance qu'il eut pour la mobilisation des associations de défense de l'environnement et, par conséquent, de la portée de Printemps silencieux pour la révolution écologiste américaine. Elle participe aussi à expliquer la virulence de ses détracteurs, qui virent dans Printemps silencieux l'expression d'un catastrophisme hystérique allant à l'encontre du progrès scientifique.

\section{L'invasion des «femelles»}

Avant même sa parution officielle, Printemps silencieux donna lieu à une campagne de communication nourrie de la part des représentants de l'industrie chimique, de scientifiques et d'hommes politiques conservateurs qui remirent en cause sa scientificité et ses méthodes et tentèrent d'en empêcher la publication ${ }^{(42)}$. Peu préparés à ce type d'attaque, les milieux industriels réagirent notamment par la parodie, à l'exemple de la nouvelle de Monsanto The Desolate Year qui déroulait le scénario catastrophe d'une invasion des États-Unis par une horde d'insectes après l'abandon total des pesticides:

«Les punaises étaient partout. Invisibles. Inaudibles. Incroyablement universelles. Au-dessus ou en-dessous de chaque lopin de terre, de chaque jardin, de chaque hectare et comté, État et région sur toute l'étendue des États-Unis. Dans chaque maison, grange, appartement et poulailler, et dans leurs charpentes, fondations et leur mobilier. À côté du sol, à côté

38 «Il ne s'agit pas de dire que les insectes ne posent aucun problème et qu'il est inutile de lutter contre eux. Je pense simplement que, d'une part, la lutte doit être menée en fonction des réalités et non d'estimations fantaisistes, et que, d'autre part, les méthodes employées ne doivent pas nous détruire en même temps que les insectes.» R. CARson, Printemps silencieux (note 3), p. 51.

39 Cf. «Printemps silencieux / Silent Spring (Carson, Rachel) », in: Dictionnaire de la pensée écologique (note 12), p. 817.

40 Sur la possible conciliation entre catastrophisme et démocratie, voir Luc SEMAL, «Catastrophisme et démocratie», in: Dictionnaire de la pensée écologique (note 12), p. 141-144.

41 Comme le DDT aux États-Unis, le Waldsterben mêla, dans les années 1980, environnement et identité nationale. Sur le Waldsterben, voir Birgit Metzger, «Erst stirbt der Wald, dann Du! D Das Waldsterben als westdeutsches Politikum (1978-1986), Francfort-sur-le-Main, Campus Verlag, 2015. 
de l'eau, sur et dans les branches, brindilles et tiges, en-dessous des rochers, dans les arbres, animaux et autres insectes - et oui, dans l'homme» ${ }^{(43)}$.

Dans cette fable apocalyptique, les insectes anthropomorphisés sont dépeints sous les traits d'ennemis féroces et sanguinaires qui, tapis dans l'ombre, attendent patiemment avant de lancer leur offensive meurtrière en déployant un arsenal de guerre savamment étudié: «[...] Certains pouvaient piquer, certains pouvaient empoisonner, beaucoup pouvaient tuer ${ }^{(44)}$. Dans une série de tableaux qui montrent la propagation croissante de la mort et de la destruction, la nature vengeresse resserre son garrot avant de connaître une dégénérescence contre-nature marquée par la prise de pouvoir d'insectes-femelles monstrueuses:

«[...] Les insectes se reproduisirent et se reproduisirent, s'hybridèrent et se croisèrent. Certains ne se reproduisirent pas du tout ou n'eurent pas à le faire; les femelles produisirent tout simplement plus de femelles qui donnèrent naissance à plus de hordes de femelles » ${ }^{(45)}$.

Sans doute la nouvelle puise-t-elle ici dans les croyances antiques selon lesquelles les colères féminines seraient du côté de la folie meurtrière, alors que la rationalité et le contrôle des émotions seraient des vertus proprement masculines. L'orientation sexuelle de Carson - la biologiste vivait en couple lesbien - n'est certainement pas étrangère à l'image d'une nature féminine pécheresse et dégénérée. Dans un espace public largement dominé par les hommes, les attaques menées contre la biologiste étaient souvent misogynes, d'autant plus que son indépendance personnelle et professionnelle en faisait une figure à contre-courant des représentations de genre traditionnelles. En pleine Guerre froide, cette misogynie s'alliait fréquemment à l'anticommunisme ${ }^{(46)}$ qui réactualisait la "peur rouge» du maccarthysme du début des années 1950 et sa politisation de l'intime à travers la figure de la femme traîtresse et perverse ${ }^{(47)}$.

À la fin de la nouvelle de Monsanto, la rationalité scientifique triomphante vient rompre le cycle infernal de la nature dégénérée et apporter le salut: après que les dix plaies d'Égypte se sont littéralement abattues sur le pays - les sauterelles dévastent

43 «The bugs were everywhere. Unseen. Unheard. Unbelievably universal. On or under every square foot of land, every square yard, every acre, and county, and state and region in the entire sweep of the United States. In every home and barn and apartment house and chicken coop, and in their timbers and foundations and furnishings. Beneath the ground, beneath the waters, on and in limbs and twigs and stalks, under rocs, inside trees and animals and other insects - and, yes, inside man." The Desolate Year, Monsanto Magazine, p. 4. Toutes les traductions sont de l'auteure.

44 «[...] Some could sting, some could poison, many could kill.» Ibid., p. 5.

45 « [...] the insects bred and re-bred, cross-bred and in-bred. Some didn't breed at all, or need to; females simply produced more females which gave birth to more hordes of females.» Ibid., p. 6.

46 Voir notamment le courrier d’un lecteur publié dans le New Yorker en 1962 ("Miss Rachel Carson’s reference to the selfishness of insecticide manufacturers probably reflects her Communist sympathies, like a lot of our writers these days. We can live without birds and animals, but, as the current market slump shows, we cannot live without business. As for insects, isn't it just like a woman to be scared to death of a few little bugs! As long as we have the H-bomb everything will be O.K.»). Dieter STEINER, Rachel Carson. Pionierin der Ökologiebewegung. Eine Biographie, Munich, oekom, 2014, p. 274.

47 L'association d'arguments antiféministes et anticommunistes était fréquente sous le maccarthysme, de même que le mythe d'une stérilisation forcée des Américains en cas de victoire des communistes. Sur la représentation iconographique de la peur pendant la Guerre froide, voir notamment J.-J. CouRTINE, «La peur, à l'âge de l'anxiété» (note 4), annexe (sans pagination). 
les plaines d'Amérique et les insectes entrent dans les villes -, la morale de l'histoire est énoncée: "Mais tous les événements majeurs de l'“année dévastée” ont bien eu lieu. [...] Ils peuvent se répéter l'année prochaine sous une forme accentuée si l'on retire à ce pays ses armes chimiques contre les nuisibles " ${ }^{(48)}$. La convocation des métaphores bibliques de l'enfer et de l'apocalypse poursuit un objectif clair: implicitement mises sur le même plan que les chiffres et analyses avancés par Carson, ces visions apocalyptiques leur renient toute crédibilité scientifique. La nouvelle se clôt par le rappel des nombreuses victoires de la chimie - à l'instar du DDT dans la lutte contre le paludisme ${ }^{(49)}$ - et l'énumération de neuf commandements pour «de meilleures années». Aux temps reculés où l'homme était soumis, impuissant, aux aléas de la nature s'oppose un hymne à la science et au progrès. Celui-ci convoque paradoxalement un scénario apocalyptique activant des «dispositifs discursifs» anxiogènes - l'ubiquité, la permanence, la nature cumulative de la menace, la confusion des temporalités - qui sont à la fois enracinés dans des temps archaïques et projetés vers l'horizon d'attente de la fin de l'humanité( ${ }^{(50)}$.

Ces deux scénarios catastrophe offrent, à des degrés d'intensité divers, un exemple de l'utilisation des peurs comme catalyseurs de l'action politique. Aussi, au lendemain de la parution de Printemps silencieux, J.F. Kennedy convoqua une commission en charge de contrôler l'utilisation des insecticides aux États-Unis. C'est toutefois près de dix ans après que Carson avait lancé son alerte que le DDT fut interdit sur l'ensemble du territoire fédéral, à l'issue de la mobilisation des associations de défense de l'environnement américaines.

\section{Le DDT dans l'espace médiatique ouest-allemand des années 1960}

Printemps silencieux et les réactions qu'il suscita illustrent l'imbrication étroite entre histoire environnementale et histoire sanitaire, croissance économique et consommation de masse, à un moment marqué à la fois par une foi forte dans le progrès scientifique et technique et par la prise de conscience naissante des limites de la croissance. Si les sociétés occidentales furent touchées par des mutations semblables, leur forme, leur ampleur et leur temporalité avaient des marqueurs nationaux. Afin de rendre compte des transferts entre les États-Unis et la République fédérale d'Allemagne, on éclairera l'évolution de la représentation et de l'interprétation des risques liés au DDT dans la presse ouest-allemande à partir d'articles parus dans l'hebdomadaire Der Spiegel ${ }^{(51)}$ au cours des années 1960 .

48 «But all the major events of the "desolate year" have actually occurred [...]. They could repeat themselves next year in greatly magnified form simply by removing this country's chemical weapons against pests.» The Desolate Year (note 43), p. 7-8.

49 "As late as 1935, there were 900,000 cases of malaria in the U.S., with 4,000 deaths. Modern insecticides, particularly DDT, have played a key role in practically stamping out the disease in this country - and in 23 others. » Ibid.

50 Cf. J.-J. Courtine, «La peur, à l'âge de l'anxiété» (note 4), p. 311-312.

51 Cette contribution se concentre uniquement sur les articles parus dans le Spiegel, même s'il ne fut bien sûr pas le seul à couvrir la parution de Printemps silencieux, à l'instar de la Zeit qui publie des extraits des $2^{\mathrm{e}}$ et $3^{\mathrm{e}}$ chapitres en été 1963. 


\section{Le DDT, une menace d'abord lointaine}

La réception de Printemps silencieux en République fédérale fut moins forte et plus tardive qu'aux États-Unis. Même si l'ouvrage d'alerte fut traduit et publié en langue allemande dès $1963^{(52)}$, la plupart des 90000 exemplaires vendus jusqu'en 1976 ne le furent que dans les années $1970^{(53)}$, dans le contexte de la réception par des figures clés du mouvement écologiste ouest-allemand des ouvrages d'alerte environnementale, à l'instar du rapport du Club de Rome sur les Limites de la croissance $^{(54)}$. Ces chiffres sont bien en deçà des ventes de l'ouvrage aux États-Unis où il avait, comme on a pu le voir, déclenché un véritable tsunami politico-médiatique. Jusque dans les années 1970, la politique et les médias ouest-allemands ne prêtèrent qu'un intérêt limité aux questions environnementales et aux questions sanitaires et toxicologiques ${ }^{(55)}$. Certes, la Détente fournit les conditions d'un double phénomène de pluralisation et de libéralisation de la société ouest-allemande: au lendemain de "l’Affaire du Spiegel», déclenchée par la publication d'un article en octobre 1962 qui avait révélé les failles de la défense fédérale, une nouvelle forme de contestation émergea; celle-ci était toutefois centrée autour de la défense des libertés et des droits fondamentaux que les suites politiques et juridiques de l'affaire semblaient remettre en question ${ }^{(56)}$. C'est dans les années 1970 et autour du mouvement anti-nucléaire, le Anti-AKW-Bewegung, que les questions environnementales firent réellement leur entrée dans l'espace public ouest-allemand.

Dans la première moitié des années 1960, les articles que le Spiegel consacre au DDT adoptent une attitude ambivalente vis-à-vis des promesses et des dangers de l'industrie chimique $^{(57)}$, à l'exemple de l'article intitulé «Lutte contre les nuisibles. Silence dans la forêt » daté de novembre 1962. Comme l'indique le titre - «Schädlingsbekämpfung» -, le journaliste caractérise le DDT et les autres pesticides auxquels Carson s'attaque dans Printemps silencieux (E 605, Aldrin, etc.) par leurs effets intentionnels, usant de qualificatifs qui mettent l'accent sur leurs effets "positifs", la protection des plantes (Pflanzenschutzmittel) et l'élimination des nuisibles, insectes (Insektenvernichtungsmittel) et mauvaises herbes (Unkrautvertilgungsmittel). Un court paragraphe rappelle en outre les "succès remarquables» («erstaunliche Leistungen») de l'"arme miracle DDT» («Wunderwaffe DDT») qui, en l'espace de dix ans, aurait permis de diviser par deux le nombre de décès liés à la malaria ${ }^{(58)}$. Reprenant les catégories d'analyse binaires des milieux industriels et des publicités de l'après-guerre, le journaliste conclut que

52 Rachel Carson, Der stumme Frühling (trad. Margaret Auer), Munich, Biederstein Verlag, 1963.

53 Voir notamment Kai Hünemörder, Frühgeschichte der globalen Umweltkrise, Stuttgart, Franz Steiner Verlag, 2004.

54 Dennis Meadows, Donella H. Meadows et al., The Limits to Growth, New York, Universe Books, 1972.

55 Voir notamment Otfried Strubelt, Gifte in Natur und Umwelt. Pestizide und Schwermetalle, Arzneimittel und Drogen, Heidelberg/Berlin/Oxford, Spektrum Akademischer Verlag, 1996.

56 «Bundeswehr: Bedingt abwehrbereit», Der Spiegel, nº 41 (1962), 10.10.1962.

57 Ce scepticisme fut encore plus prononcé en France, où la presse réserva un accueil sceptique aux thèses de Carson. Cf. A. Trespeuch-Berthelot, «La réception des ouvrages d'alerte environnementale dans les médias français» (note 7), p. 9. Cf. «Schädlingsbekämpfung. Schweigen im Walde» (note 8). 
l'Alliance fédérale pour l'industrie chimique pourrait légitimement parler de «l'un des chapitres les plus honorables et les plus actuels de l'histoire mouvementée de la chimie ${ }^{(59)}$. À l'intérieur d'un large panorama des enjeux soulevés par l'ouvrage d'alerte et des réactions qu'il suscita, le journaliste interroge longuement le potentiel de toxicité des pesticides, en faisant le catalogue des principales observations de Carson qui, présentée comme «Dr. Rachel Carson», apparaît sous les traits d'une autorité scientifique qualifiée et de renom. S’ils sont questionnés, les risques sont placés sous le signe de l'incertitude et de l'éloignement spatial et temporel. Faisant un usage répété de la modalité, le journaliste indique que Carson dépeint « [...] ce qui pourrait un jour arriver aux habitants de la terre: la mort par empoisonnement via la pollution intentionnée et systématique de la nature par les produits chimiques ${ }^{(60)}$. L'alerte de Carson concerne alors prioritairement la nation américaine que ce «scénario d'horreur» a plongé «dans l'effroi " (61). Dans cet article principalement informatif, la distance spatio-temporelle se double d'une distanciation émotionnelle: le temps est celui du constat relativement neutre - le journaliste présente les craintes de Carson comme étant «crédibles » («plausibel») et «compréhensibles» («verständlich») ${ }^{(62)}$ - et non de l'alerte.

\section{Un scandale peut en cacher un autre - parallèles entre le Contergan et le DDT}

La terminologie et la tonalité du Spiegel sont plus incisives dans un article qui reproduit une interview avec le directeur du groupe pharmaceutique Bayer, Heribert Wilmes. Un court encadré rappelle le développement exponentiel que connut le marché des pesticides en République fédérale : avec près de 80 insecticides commercialisés par le groupe, parmi lesquels le E 605, le chiffre d'affaires dans ce secteur était alors sur le point de dépasser celui des produits pharmaceutiques ${ }^{(63)}$. Comme l'indique le titre de l'interview - «Où la guerre chimique totale nous mène-t-elle?» -, la menace de la "guerre totale» menée contre les «nuisibles» est placée sous les auspices du passé traumatique de la "guerre totale» nationale-socialiste et de l'avenir redouté d'une Troisième Guerre mondiale. Aussi, ne plaidant pas pour l'interdiction totale des pesticides mais pour leur emploi plus mesuré, les journalistes se font les relais des peurs liées à l'utilisation massive des pesticides dans la continuité du débat virulent déclenché par la parution de «l'ouvrage d'alerte polémique» de Carson ${ }^{(64)}$. Dénonçant le marketing

59 «Diese Taten legitimieren durchaus, von "einem der stolzesten und aktuellsten Kapitel in der ereignisreichen Geschichte der Chemie” zu sprechen, wie es der 'Verband der Chemischen Industrie' in der Bundesrepublik erst kürzlich tat. »Ibid., p. 119.

60 «In ihrem soeben veröffentlichten Buch [...] malt die renommierte Meeresbiologin und BestsellerAutorin [...] aus, was den Erdbewohnern dereinst widerfahren könnte: der Gifttod durch die selbstgewollte, systematisch betriebene Verseuchung der Natur mit Chemikalien.»Ibid. Toutes les mises en exergue en italiques sont de l'auteure.

61 «Mit diesem erfundenen Horror-Porträt einer amerikanischen Kleinstadt hat die Wissenschaftlerin Dr. Rachel Carson die US-Nation in Schrecken versetzt.» Ibid.

62 Ibid., p. 120.

63 " "Wohin führt der totale Giftkrieg?” Spiegel-Gespräch mit Bayer-Direktor Heribert Wilmes », Der Spiegel, $\mathrm{n}^{\circ} 42$ (1964), 14.10.1964.

64 «Bayer-Direktor Wilmes [...] verschließt sich der Problematik nicht, die mit der Massenausbringung chemischer Insektizide verknüpft ist und die vor allem seit Erscheinen des polemischen Mahn-Buches "Der stumme Frühling” von Rachel Carson in der Öffentlichkeit heftig diskutiert wird [...]. » Ibid., p. 71. 
de Bayer et son manque de transparence, les journalistes s'érigent en porte-parole des victimes potentielles, établissant une analogie avec le scandale du Contergan, auquel le Spiegel avait consacré sa une en mars $1963^{(65)}$ :

«Veut-on attendre que les dommages contre lesquels on met aujourd'hui en garde se réalisent? Veut-on attendre qu'il se passe quelque chose de semblable à ce qui s'est passé dans le cas du Contergan?» ${ }^{(66)}$.

Commercialisé en République fédérale depuis 1957 par le laboratoire rhénan Grünenthal, le somnifère et calmant avait été retiré du marché en novembre 1961 après la révélation de milliers de cas de fausses couches et de malformations congénitales de foetus suite à la prise du médicament par des femmes enceintes. Longtemps associé au mythe de la «catastrophe originelle»(Urkatastrophe) ${ }^{(67)}$, ce scandale avait provoqué un choc profond dans l'opinion publique ouest-allemande, questionnant les pratiques des industriels, les marges de manœuvre des administrations publiques et les normes juridiques, ainsi que leur impact sur les consommateurs, et en l'occurrence les consommatrices. Participant au phénomène de libéralisation de l'espace public et à la remise en cause du monopole de l'information par les autorités publiques et les industriels ${ }^{(68)}$, ce scandale s'inscrivait dans un contexte sociétal plus large qui interrogeait la gestion de l'incertitude et la nécessaire adaptabilité des politiques de prévention. Le parallèle établi entre le Contergan et le DDT illustre la stratification des peurs dans une société ouest-allemande sensibilisée aux risques - notamment à celui du Fallout nucléaire - et dans laquelle la question des responsabilités (individuelles et collectives) et des preuves statistiques occupait d'autant plus le devant de la scène publique dans le contexte des procès d'Auschwitz (1963-1968).

Les parallèles que l'on observe entre les États-Unis et la République fédérale dans la perception des risques liés aux pesticides se retrouvent également du côté des industriels: sans nier les risques encourus, Wilmes tente de désamorcer la situation, articulant son propos autour de trois idées forces qui font écho aux arguments de Monsanto. Premièrement, l'opposition entre objectivité scientifique et catastrophisme irrationnel. Printemps silencieux serait le «symptôme de l'inquiétude actuelle» ${ }^{(69)}$ et Carson l'un de ces «alerteurs immenses» («übergroßen Warner», p. 79) qui exagèrent les faits

65 Cf. «Kopfweh wird mitgeliefert», Der Spiegel, n 10, 06.03.1963, p. 44-61.

66 «Will man denn warten, bis jene Schäden, vor denen heute gewarnt wird, eingetreten sind? Will man warten, bis sich etwas Ähnliches ereignet wie beim Contergan?» "Wohin führt der totale Giftkrieg?» (note 63). Le Spiegel reprend la formulation de "guerre chimique totale» («totaler Giftkrieg») employée par le scientifique Hermann J. Muller.

67 Cf. Nils Kessel, «Contergan in der Konsumgesellschaft. Wissen und Nichtwissen über Arzneimittelverbrauch in der Bundesrepublik, 1955-1962», in: Thomas GroßBöLting, Niklas LENHARDSснгамm (éd.), Contergan. Hintergründe und Folgen eines Arzneimittel-Skandals, Göttingen, Vandenboeck \& Ruprecht, 2017, p. 71.

68 Sur ce point, voir notamment l'étude de Niklas Lenhard-Schramm qui offre un aperçu des dimensions politiques, juridiques et sociétales du scandale: Niklas Lenhard-Schramm, Das Land NordrheinWestfalen und der Contergan-Skandal. Gesundheitsaufsicht und Strafjustiz in den "langen sechziger Jahren », Göttingen, Vandenhoeck \& Ruprecht, 2016.

69 «Das Buch ist sicher ein Symptom für eine Unruhe, die da ist. Aber was wir ablehnen, sind die Vereinfachungen, Übertreibungen und Verzerrungen, die - neben einigen Fakten - in dem Carson-Buch enthalten sind.» «Wohin führt der totale Giftkrieg?» (note 63), p. 71. 
pour accroître leur audience. Deuxièmement, l'assimilation de ces peurs à un état de trouble intérieur ( Unnruhe»), naïf et passéiste:

«Il y a toujours eu des choses au sujet desquelles les hommes se sont inquiétés. Quand j'étais élève, on disait que les tomates étaient responsables du cancer, puis les engrais, puis les marmites en aluminium. Madame Tout-le-monde ne voulut plus rien cuisiner dans la marmite en aluminium. Je trouve sympathique que l'être humain s'inquiète et s'intéresse à beaucoup de choses. Je ne peux malheureusement rien changer au fait que le progrès comporte aussi ses inconvénients» ${ }^{(70)}$.

En rapportant la question des risques à la psychologie des temps modernes, Wilmes les détourne de leur substrat objectif et matériel, élude la question de leur exceptionnalité et de la responsabilité des industriels ${ }^{(71)}$. Populaires et féminines - comme le montre la référence à la «marmite en aluminium» de "Madame Tout-le-monde»-, ces croyances s'opposent à l'expertise (masculine) des industriels et aux nombreux tests réalisés à la recherche d'effets secondaires. Troisièmement, l'inscription des enjeux environnementaux et sanitaires dans un espace privé dépolitisé. Alors que l'engagement des "passionnés d'oiseaux» et des "amis des animaux» est assimilé à un simple loisir ${ }^{(72)}$, la décision de consommer des produits traités relèverait de critères personnels de confort et d'esthétique et non du domaine de la santé publique: rappelant que sa famille consomme chaque Noël «avec grand appétit» des «[...] pommes issues de la ferme Bayer sur lesquelles de nouveaux herbicides ont été testés» et qui auraient sans doute une piètre apparence si elles ne l'avaient pas été ${ }^{(73)}$, Wilmes dénie à la question des risques toute pertinence politique.

\section{De la menace latente au risque réel - la «catastrophisation» du discours}

Les lignes de conflits entre différents intérêts (économiques, environnementaux et sanitaires) et temporalités (effets à court et à long terme) se renforcent au tournant des années 1960-1970. Elles s'inscrivent dans le contexte de la démultiplication d'études alarmistes sur le DDT et de l'augmentation des restrictions et interdictions dans le monde ${ }^{(74)}$. Outre l'augmentation quantitative du nombre d'articles consacrés au DDT, cela se traduit

70 «Und es gab wohl immer etwas, worüber die Menschen sich beunruhigt haben. In meiner Pennälerzeit hieß es noch, die Tomaten seien schuld am Krebs, dann waren es die Düngemittel, dann die Aluminiumtöpfe. Tante Emma wollte nichts mehr aus dem Aluminiumtopf haben. Ich finde es auch sympathisch, daß der Mensch unruhig ist und sich für vieles interessiert. Daß der Fortschritt auch Nachteile hat, das läßt sich nun leider nicht ändern.» Ibid., p. 81.

71 Cet argument rappelle les théories de la dépendance qui circulèrent dans les milieux psychiatriques suisses des années 1950 expliquant la dépendance aux somnifères et aux antidouleurs par la structure de la personnalité des patients («persönlichkeitszentriertes Suchtparadigma»). Voir N. KEssEL, «Contergan in der Konsumgesellschaft» (note 67), p. 79.

72 «Es gibt Leute, Vogelliebhaber und Tierfreunde - ich habe auch meine Liebhabereien, und wenn man da irgend etwas wittert, wird man natürlich unruhig und übertreibt auch, damit die Öffentlichkeit aufmerksam wird.» "Wohin führt der totale Giftkrieg?» (note 63), p. 72.

73 «Ja, also, das stört mich persönlich nicht im geringsten. Meine Familie bekommt jedes Jahr um die Weihnachtszeit hier vom Bayer-Versuchsgut einen halben Zentner Äpfel, an denen neue Pflanzenschutzmittel ausprobiert worden sind - wir essen das mit größtem Appetit. Wenn sie nicht gespritzt worden wären, sähen sie wahrscheinlich schlimm aus.»Ibid. 
par une dramatisation de la terminologie employée dans les articles de l'hebdomadaire ouest-allemand:

«Le poison est partout. Il contamine les nappes phréatiques, pollue les fleuves, retombe sur terre avec la pluie, se propage dans les océans. Aucune région de la terre n'est épargnée récemment, il s'est propagé jusque dans les régions polaires. Les hommes et les animaux l'ingèrent involontairement dans leur nourriture. Jusqu'à présent, il passait pour être inoffensif pour les hommes et les animaux plus développés - il n’est immédiatement mortel que pour les insectes: le pesticide DDT ${ }^{(75)}$.

Dans cet incipit tiré de l'article "Interdiction du DDT. Une couvée contaminée» daté de juin 1969, les risques liés au DDT ne sont plus présentés sous les traits d'une sombre vision d'avenir, mais comme un fait avéré, ce qui est marqué par l'utilisation du présent et de courtes phrases qualificatives. Après avoir présenté différentes «catastrophes liées au DDT» («DDT-Katastrophen»), l’article déploie la vision catastrophiste d'un poison insidieux («Gift», «DDT-Vergiftung», "DDT-Giftwirkungen») qui a envahi tous les recoins de la terre et des océans. L'accent ne porte plus sur le produit lui-même en tant qu'insecticide, mais sur son interdiction: la menace latente a cédé la place au risque réel inscrit dans une permanence temporelle qui englobe les échelles locale et globale. Ce catastrophisme s'accentue encore au mois de décembre 1969, quand le Spiegel publie une interview avec la ministre de la Santé Käte Strobel: dans un court article introductif intitulé «DDT. Chaîne dangereuse », le journaliste indique que «[d]ans les derniers mois se sont multipliés les signes que les sombres visions d'avenir de Rachel Carson pourraient devenir réalité ${ }^{(76)}$. Le «retard» des pouvoirs publics ouest-allemands est pointé du doigt: au temps de l'urgence - le journaliste parle de «bombe à retardement biologique» («biologische Zeitbombe») - s'oppose le temps long des tests scientifiques, des contrôles et du processus législatif - à l'instar de la loi sur les pesticides ("Giftgesetz») en cours d'élaboration par le ministère de la Santé(77). Intitulé «Le risque est certainement indéniable», cet article ne laisse plus de place au doute: à la globalisation des risques répond la globalisation des peurs.

Aussi, peu de temps avant, le 24 novembre 1969, le Spiegel avait-il publié le scénario apocalyptique du professeur américain Paul Ehrlich $^{(78)}$ : dans cette dystopie, les océans meurent et la pêche s'effondre au fil de l'exploitation incontrôlée des ressources par l'homme et de la survenue de plusieurs catastrophes environnementales. Comme Carson, Ehrlich s'appuie sur les évolutions de son époque pour les amplifier, tout en

75 «Das Gift ist überall. Es durchsetzt das Grundwasser, verpestet die Flüsse, tröpfelt mit dem Regen zur Erde, verteilt sich in den Weltmeeren. Kein Gebiet auf der Erde bleibt von ihm verschont - jüngst ist es bis in die Polregionen vorgedrungen. Menschen und Tiere konsumieren es mit der Nahrung als unfreiwillige Zukost. Bislang galt es als harmlos für Menschen und höher entwickelte Tiere - nur bei Insekten wirkt es sofort tödlich: das Schädlingsbekämpfungsmittel DDT.» «DDT-Verbot - Verseuchte Brut», Der Spiegel, nº 24 (1979), 09.06.1969, p. 162.

76 «In den letzten Monaten häuften sich die Anzeichen, daß Rachel Carsons düstere Vision Wirklichkeit werden könnte. » «DDT. Gefährliche Kette», Der Spiegel, nº 50 (1969), 08.12.1969.

77 Cf. «Das Risiko ist sicher unbestritten», ibid. C’est en août 1972 («Gesetz über den Verkehr mit DDT») que le DDT fut interdit en République fédérale.

78 Cf. «Wir sind dabei, den Planeten Erde zu ermorden. Umweltverseuchung bis zum Jahre 1980 / von Dr. Paul Ehrlich », Der Spiegel, nº 48 (1969), 24.11.1969. 
adoptant une tonalité plus catastrophiste, conjurant le «viol et le meurtre de [la] planète par simple profit $[. ..]{ }^{(79)}$ : après que les océans et la terre sont devenus hostiles à la vie, les populations sont tour à tour décimées par la famine et les épidémies. Mêlant enjeux sanitaires et géopolitiques, ce scénario apocalyptique entraîne l'humanité entière dans une guerre mondiale nucléaire.

\section{Conclusion}

«[La] modernité, qui portait depuis les Lumières la promesse de faire advenir un monde où les peurs anciennes seraient dissipées, a accouché d'un âge de l'anxiété $[\ldots]{ }^{\left({ }^{(80)}\right.}$. Rappelant que les sociétés du $\mathrm{XX}^{\mathrm{e}}$ et du début du $\mathrm{XXI}^{\mathrm{e}}$ siècles ont fait l'expérience de désastres multiples dans un monde toujours plus globalisé, cette analyse permet d'éclairer les enjeux des discours et contre-discours sur le DDT qui se diffusèrent dans les espaces publics américain et ouest-allemand dans l'après-guerre. Inscrits, en ces débuts de Guerre froide, à l'intérieur d'une vision binaire du monde, ces discours représentent deux facettes de la modernité technique et scientifique, placée tantôt sous le signe du progrès et de la finalité ultime de l'humanité, tantôt sous celui de la destruction de la planète par l'homme. La mise en parallèle de ces débats offre un exemple de la circulation des peurs et de leurs différentes stratifications (la peur du manque de ressources, de la guerre et des épidémies) et de l'angoisse liée à la crainte d'une guerre nucléaire destructrice. La «catastrophisation» croissante des images et des discours dans l'hebdomadaire ouest-allemand Der Spiegel témoigne du renforcement et de la globalisation des risques et des émotions à l'aube des années 1970. Paru il y a plus de cinquante ans, l'ouvrage de Rachel Carson et les questionnements qu'il soulève sont toujours d'actualité: figure d'un temps et d'une culture donnés, Rachel Carson semble préfigurer le ton péremptoire de Greta Thunberg quand elle dit que «[1]es générations futures nous reprocheront probablement de ne pas nous être souciés davantage du sort futur du monde naturel, duquel dépend toute vie ${ }^{(81)}$. Il est frappant de constater que, si les problèmes dénoncés par Carson demeurent - en témoignent les débats récents autour du glyphosate -, les critiques adressées aux alerteurs et $a$ fortiori aux alerteuses, plus facilement soupçonnées d'hystérie catastrophiste, perdurent. Honnies autant qu'adulées, ces deux alerteuses utilisent la peur comme mode d'action politique, sans toutefois sombrer dans un discours apocalyptique dénué de tout sens de la réalité. En cela, elles posent la question du difficile équilibre entre catastrophisme et démocratie.

79 «Die Gesellschaft der westlichen Welt ist in der Tat im Begriff, die Vergewaltigung und den Mord unseres Planeten aus Gewinnstreben zu vollenden.»Ibid., p. 201.

80 J.-J. Courtine, «La peur, à l’âge de l'anxiété» (note 4), p. 313.

81 R. Carson, Printemps silencieux (note 3), p. 55. Voir notamment l'essai paru récemment dans la Zeit qui met en exergue les parallèles entre le combat des deux femmes. Cf. «Umweltschutz, Die Macht des Individuums», Die Zeit, 15.09.2019 (consulté le 15.09.2019). 


\title{
Résumé
}

Publié en 1962, Printemps silencieux est considéré comme l'un des déclencheurs de la révolution verte aux États-Unis. Dans cet ouvrage, la biologiste américaine Rachel Carson met en garde ses contemporain.e.s contre l'empoisonnement de la nature et des êtres vivants en raison de l'utilisation massive de pesticides, parmi lesquels le DDT. Prenant la forme d'une fable scientifique, son plaidoyer en faveur d'un changement nécessaire des modes de pensée et d'action a suscité de vives réactions de la part d'hommes politiques conservateurs, de scientifiques et d'industriels, qui l'ont accusée d'hystérie catastrophiste et d'ignorance (féminine), y opposant les promesses du progrès scientifique et technique. Cet article s'intéresse aux discours et contre-discours sur le DDT dans l'après-guerre et questionne leur radicalité à l'aune du concept de catastrophisme. L'étude d'une série d'articles publiés dans l'hebdomadaire ouest-allemand Der Spiegel dans les années 1960 montre l'émotionalisation du débat sur les questions environnementales et sanitaires tout en révélant les stratifications multiples et la transnationalité de la peur pendant la Guerre froide.

\section{Zusammenfassung}

In ihrem 1962 erschienenen Buch Der stumme Frühling, das als Zünder der USamerikanischen grünen Bewegung gilt, warnt die Biologin Rachel Carson ihre Mitmenschen vor der Vergiftung der Natur und des Menschen durch den massiven Einsatz von Pflanzenschutzmitteln, an erster Stelle des Insektizids DDT. Carsons Plädoyer für einen notwendigen Wandel der Denk- und Handlungsweisen, das sie in eine wissenschaftlich fundierte Fabel einfügt, rief heftige Kritik bei konservativen Politikern, Wissenschaftlern und Industriellen hervor, die ihr hysterische Panikmache und (weibliches) Unwissen vorhielten und den technischen und wissenschaftlichen Fortschritt hochpriesen. Dieser Artikel konfrontiert die Diskurse, Motive und Strategien beider Seiten und hinterfragt ihre gesellschaftliche Anschlussfähigkeit bzw. Radikalität anhand des Katastrophismus-Begriffs. Das Heranziehen von ausgewählten Spiegel-Artikeln der 1960er Jahre zeigt die emotionale Aufladung der Debatten über Umwelt- und Gesundheitsprobleme und gibt Einblicke in die transnationale Dynamik und Vielschichtigkeit des Angstdiskurses im Kalten Krieg.

\begin{abstract}
Rachel Carson's Silent Spring (1962) is regarded as having triggered the green movement in the USA. Carson warns against the massive use of pesticide, among which the insecticide DDT, that leads to poisoning of nature and living beings. Her plea for change in the way we think and act takes the form of a scientifically-based tale. It sparked fierce criticism from conservative politicians, scientists and industrialists, who accused her of hysterical panic-mongering and (female) ignorance while simultaneously praising technical and scientific progress. This article studies the discourses, motives and strategies of both sides and questions their radicalism through the prism of the concept of catastrophism. The comparison with selected Spiegel articles from the 1960 s stresses the emotionalization of the debates on environmental and health problems while providing insights into the transnational dynamics and complex stratifications of fear during Cold War.
\end{abstract}

